\title{
Development of Biopsy Needle Design and Optimum System
}

\author{
Tuodang Guo ${ }^{1,2, a}$, Qinhe Zhang ${ }^{1,2, b^{*}}$, Lei $\operatorname{Tan}^{1,2, c}$, Guowei Liu ${ }^{1,2, d}$, \\ Hongjian Dong ${ }^{1,2, \mathrm{e}}$ \\ ${ }^{1}$ School of Mechanical Engineering, Shandong University, Jinan 250061, China \\ ${ }^{2}$ Key Laboratory of High Efficiency and Clean Mechanical Manufacture of Ministry of Education, \\ Shandong University, Jinan 250061, China \\ aguo804603577@163.com, ${ }^{\mathrm{b}}$ zhangqh@sdu.edu.cn, '9ei_tan7@hotmal.com, \\ Igw52008@foxmail.com, edonghongjianzz@163.com
}

Keywords: secondary development, design and optimum system, ABAQUS, biopsy needle, plane surface, cylindrical surface.

Abstract: A sharp edged biopsy needle is often used to cut and remove the expected tissue specimen to identify and diagnose cancer and other diseases. However, the traditional way to design and systematicness. The efficience of designing and optimizing of biopsy needle can be improved by selecting appropriate needle tip geometry. In this paper, a design and optimum system of biopsy needle is developde with the help of the secondary development of ABAQUS, by which the plane and cylindrical surface biopsy needle model can be automatically established through inputting the parameters of the needle tip. The plane surface biopsy needles were contrasted with cylindrical surface biopsy needles in rake and inclination angle by MATLAB. The results show that the cylindrical surface needle tip had a broader distribution in the rake and inclination angle than one-plane needle. Therefore, the cylindrical surface needle may be more conducive to the biopsy.

\section{Introduction}

Sampling is a key step in the process of biopsy or many other health care procedures, in whcih the common used device to cut and remove the tissue specimen is biopsy needle in the clinical. The data from the clinical pratice shows that the failure rate captured any tissue is up to $27 \%$ [1]. It is necessary to improve the success rate of the biopsy needle. The cutting edge geometry of biopsy needle plays an important role in the result of biopsy and the efficiency of sampling.

Some research has been done to study cutting edge geometry of biopsy needle. Jason et al [2-3] found that a large rake and inclination angle can reduce the penetration force and obtain a better penetration performance by establishing a mathematical model of the plane and curved surface needle tip. Ehmann and Malukhin [4] analyzed the distribution of the rake and inclination angle along the biopsy needle tip and applied it to the plane needle, cylindrical surface needle and screwed surface needle. However, they did not take the systematic design and optimum of biopsy needle into consideration, which plays an important role in the improvement of efficiency of biopsy.

Since only little needle tip geometry is used in the clinical applications, the traditional way to design and produce needles is always tentative, which involves little needle tip geometry and lacking of systematicness. In this paper, the secondary development of ABAQUS for automatic parameterized modeling of the plane and cylindrical surface needle was been carried out, and the rake and inclination angle were calculated by the MATLAB. The system can increase the speed of creating the needle tip model, reduce duplication of modeling steps and develop a systematic process of the design and optimum of the biopsy needle tip geometry. What's more, it can provide a reference for the development of the new style needle.

\section{Design and optimum system developed by ABAQUS}

A new script and graphical user interface were created in the design and optimum system, which can realize the finite element analysis automated processing of biopsy needle modeling through a GUI Toolkit. The scripting interface of ABAQUS was customization and developed based on the 
Python language. Python is an object-oriented high-level programming language, which is easy to understand. Graphical interface is a convenient tool for user to input or select parameters, of which ABAQUS/CAE is a typical GUI program. The main solving process is shown in Fig. 1(a).

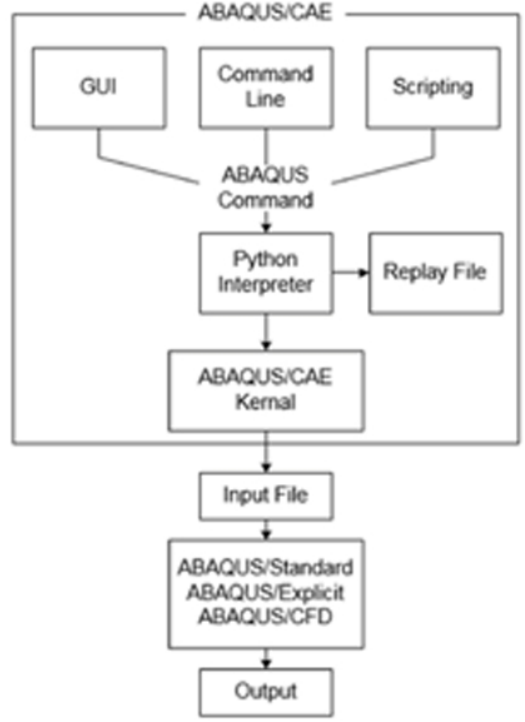

(a)

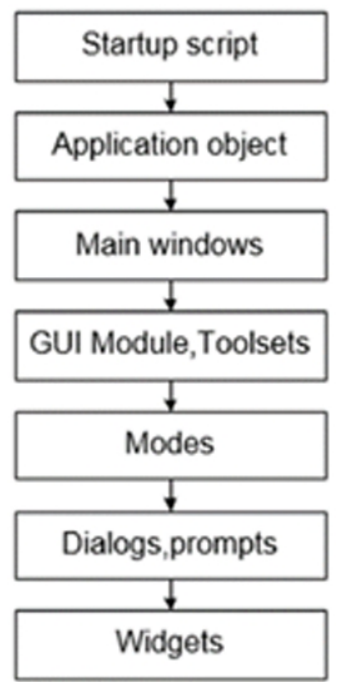

(b)

Figure 1 The process of solving(a) and GUI structure(b)

The graphical user interface-GUI (for example, ABAQUS/CAE) can complete the interaction function between human and computer by interaction between the kernel and GUI. Kernel program contains the all object data and methods of ABAQUS/CAE, which can carry out various operations by the data and methods, such as create components and mesh. By the internal communication protocol program, GUI sends the kernel command to kernel program. Then, the kernel command is explained and executed by the kernel program.

The design and optimum system of biopsy needle is a custom-designed GUI interface (Fig. 2(b)). The system application is called by the startup script. Then, the system main window displays, the system main interface structure is defined, including menus, toolbars etc. The system window would be loaded, including the specified module and tool sets. The different menus and buttons correspond to different modes which refer to particular GUI response mechanism, control the display of a dialog box, and assign a particular button and instruction. The widgets in the dialog are used to implement the interaction between user and system, such as input data, trigger commands and call by ABAQUS/CAE kernel.

The whole structure of this system is constituted by procedures, main interface window, toolsets, and corresponding a number of modes. The load and start implemented by a function call step by step. The system interface as shown in Fig. 2(a).

The design of plane and cylindrical surface needle has completed by the design and optimum system of biopsy needle. The plane needle includes one-plane needle, symmetric and asymmetric two-plane needle, and symmetric three-plane needle. The plane needle mainly introduced the one-plane needle, as shown in Fig. 2(b). This dialog is the automatically parameterized modeling interface of the one-plane needle by inputting the parameters of the plane cutting angle, inside and outside radius. When modified these parameters, one-plane model can be automatically generated, as shown in Fig. 2(c). 


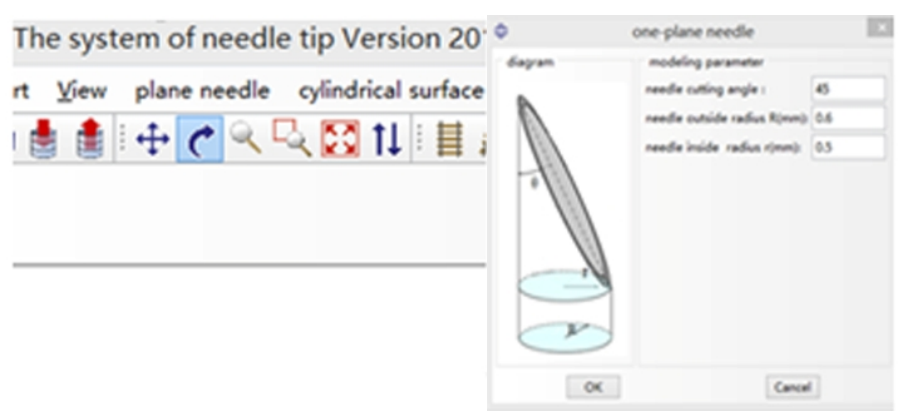

(a) (b)

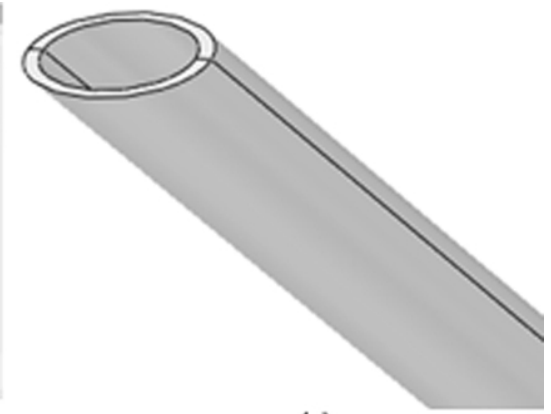

(c)

Fig 2 The needle system interface (a) and the one-plane needle(b, c)

The cylindrical surface needle automatically parameterized modeling interface is shown in Fig. $3(a)$, which contains XY plane unit function $\left(y=(x-1)^{2}\right.$ by default $)$, inside and outside radius, and the value of $y$ calculated according to the unit function. When inputting the parameters, the cylindrical surface needle model can be built, as shown in Fig, 3(b).

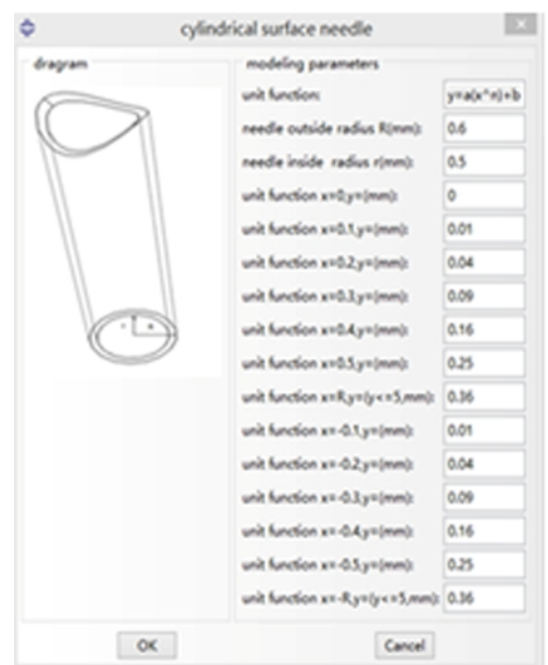

(a)

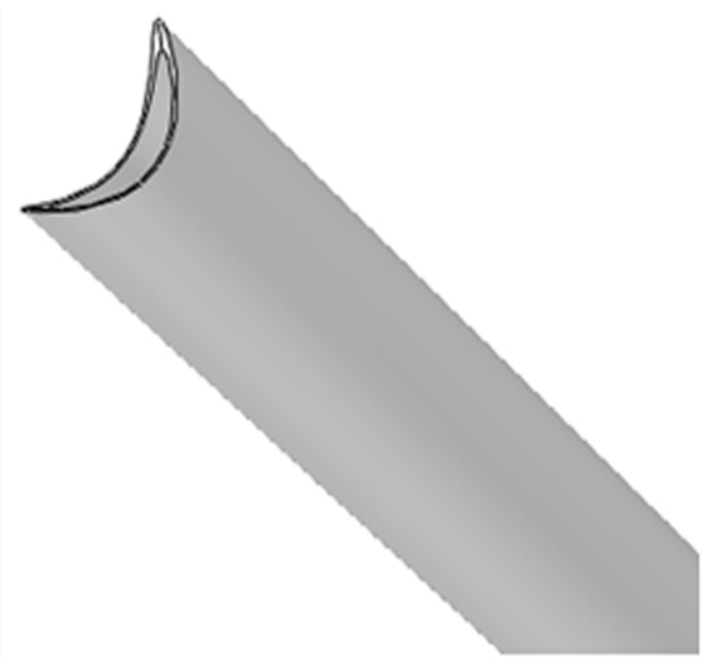

(b)

Fig 3 The cylindrical interface (a) and the cylindrical needle (b)

\section{Cutting edge geometry of biopsy needle}

The cutting edge geometry is very important to the process of puncture for analyzing the tissue deformation and the needle bending, which can be characterized by the inclination angle $(\lambda)$ and rake angle $(\alpha)$, it has been showed that when the inclination angle is small enough it is easy to cause the failure of puncture tissue. Therefore, understanding the needle tip geometry parameters $\lambda, \alpha$ is very important to reduce the puncture force. In order to analyze cutting edge geometry of biopsy needle, one coordinate system was established, as shown in Fig. 4(a). The parameter equation of cutting edge is [5]:

$$
\left\{\begin{array}{c}
x(\gamma)=r * \cos \gamma \\
y(\gamma)=r * \sin \gamma \\
z(\gamma)=r(1-\cos \gamma) * \cot \xi
\end{array}\right.
$$

The any point in the cutting edge geometry can be characterized by the rake angle $(\alpha)$ and inclination angle ( $\lambda$ ). The $\alpha$ and $\lambda$ are solved by Moore et al [2], as expressed in Eq. (2) and Eq. (3).

$$
\alpha=\arccos \sqrt{\sin ^{2} \gamma+\cos ^{2} \gamma \cos ^{2} \gamma}
$$




$$
\lambda=\arcsin \frac{|\cot \xi * \sin \gamma|}{\sqrt{1+\cot ^{2} \xi e \cot ^{2} \gamma}}
$$

Where, $\xi, r, \gamma$ are the needle bevel angle, radius, and the angle of specific point, respectively. They are constant when involving a given needle.

The cylindrical surface needle is a simple and basic type of curved surface needle .The cylindrical surface could be formed by one or more surfaces constructed by a two-dimension line which is extruded in a linear direction. In order to analyze the cylindrical surface needle, other coordinate system is established, as shown in Fig. 4(b). The parameter equation of cutting edge is [3]:

$$
\begin{aligned}
& x=r \cos \gamma \\
& y=r \sin \gamma
\end{aligned}
$$

$\mathrm{z}=$ any continuously differentiable equation

The $\alpha$ and $\lambda$ are also solved by Moore et al [2], as expressed in Eq. (5) and Eq. (6).

$$
\begin{aligned}
& \lambda=\arcsin \frac{\left|\mathrm{x}^{3}(\mathrm{r})\right|}{\sqrt{\mathrm{r}^{2}+\mathrm{z}^{3}(\mathrm{r})^{2}}} \\
& \alpha=\arccos \frac{r^{2}+z^{\prime}(r)^{2}}{\sqrt[r]{r^{2}+z^{\prime}(r)^{2}\left(2+\cot ^{2} r\right)+\left[\frac{\left.z^{\prime}\left(r^{3}\right)^{4}\right]}{r^{2} \sin ^{2} r}\right]}}
\end{aligned}
$$

Where, $r$ is the cylindrical surface needle radius.

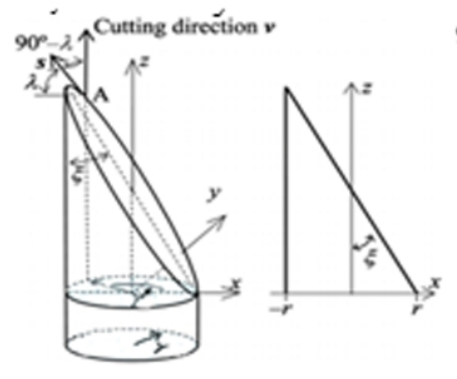

(a)

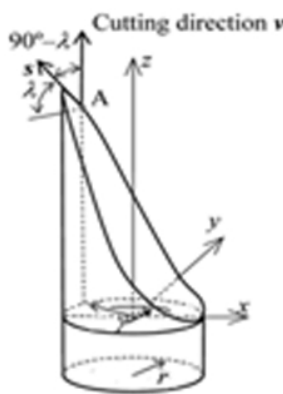

(b)

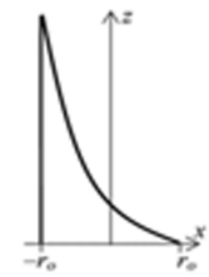

Figure 4 Bias bevel needle model (a) and cylindrical surface needle model (b)

According to the rake and inclination angle formula of the needle, the one-plane (Fig. 5(a,b)) and cylindrical surface needle $\left(y=(x-1)^{2}\right.$, Fig. 5(c). ) were calculated by the MATLAB. The result of the Fig. 1 indicated that the cylindrical surface needle tip has a broader distribution than one-plane needle in the rake and inclination angle.

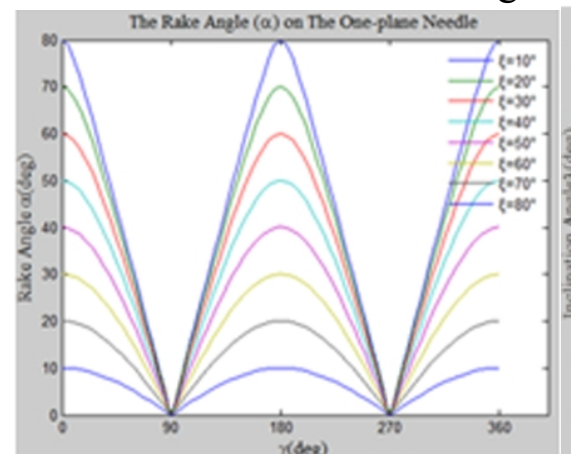

(a)

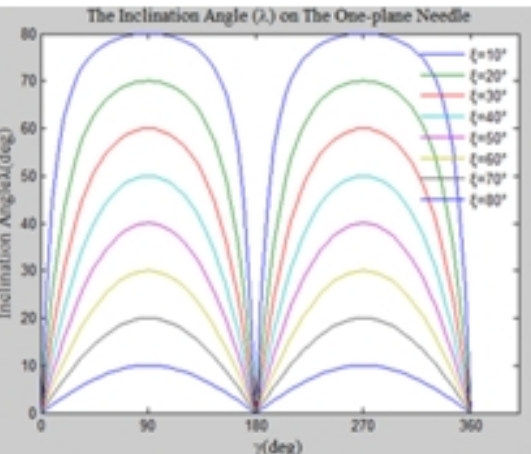

(b)

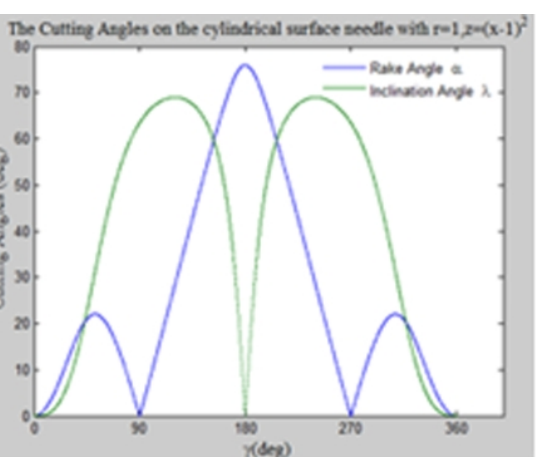

(c)

Fig 5 The rake and inclination angle on the one-plane needle $(a, b)$ and cylindrical surface needle(c) 


\section{Conclusion}

A design and optimum system of biopsy needle is developed by the secondary development of ABAQUS in this paper, which can realize the design and optimum of the plane needle and cylindrical surface needle tip geometry. The one-plane needle and cylindrical surface needle were contrasted by MATLAB, the result shows that the cylindrical surface needle tip has a broader distribution in the rake and inclination angle than one-plane needle. Therefore, the cylindrical surface needle may be more suitable for the biopsy. The design and optimum system of biopsy needle can improve the efficiency for design the needle tip geometry by reducing a lot of modeling steps. Also, in the design and producing of biopsy needles, the system has a more systematic way compared with the traditional method by automatic constructing model. The system can provide reference for the design of the complex curved surface needle in the subsequent work.

\section{Acknowledgements}

The work is supported by grants from The National Natural Science Foundation of China (Grant No. 51475274) and The Specialized Research Fund for the Doctoral Program of Higher Education ( Grant No. 20130131110070).

\section{References}

[1] G. Ubhayakar, W. Li, C. Corbishley, U. Patel, Improving glandular coverage during prostate biopsy using a long-core needle: technical performance of an end-cutting needle, BJU Int. 89(1) (2002), pp.40-43.

[2] J. Z. Moore, Q. Zhang, et al., Modeling of the plane needle cutting edge rake and inclination angles for biopsy. J. Manuf. Sci. Eng. 132(5) (2010), pp. 1-8.

[3] J. Z. Moore, Q. Zhang, et al. Modeling cutting edge geometry for plane and curved needle tips. Proc I Mech Part B: J Engineering Manufacture. 226 (2012) pp.861-869.

[4] K. Ehman, K. Malukhin, A generalized analytical model of a biopsy needle tip. J. Manuf. Sci. Eng- ASME, 134(6) (2012) 061001.

[5] L. Guo, Q. Zhang, M. Liu, Indentation test of soft tissue for investigating needle tissue machining. MACE, (2011) pp. 348-351. 